\title{
Breaking Down Silos: Interprofessional Collaborative Practice in Humanitarian Audiology
}

\author{
Lindsay M. Bondurant, Ph.D. ${ }^{1}$
}

\section{ABSTRACT}

The goal of humanitarian healthcare is to improve health outcomes and patient quality of life in under-resourced areas. One avenue for improvement may be via interprofessional collaborative practice, which allows providers from multiple specialties to work together to promote positive interventions for the communities they serve. The purpose of this article is to provide a general framework for incorporating interprofessional collaborative practice within a humanitarian audiology project.

KEYWORDS: Healthcare, silos, humanitarian audiology, interprofessional collaborative practice

\begin{abstract}
Audiologists are fortunate to have the privilege of working with people and have a positive impact on the world; for many, this was the primary reason to work in this field. Audiologists spend time evaluating hearing, managing hearing loss, as well as balancing disorders; and along the way, get to connect to patients and their families in ways that enrich their own lives in addition to those of the people they serve. Hearing healthcare providers are trained to view patients holistically, as human beings with a complex history and communication needs that shift and change in various situations. As allied health professionals, audiologists must skillfully navigate interactions with other pro-
\end{abstract}

fessionals while striving to improve patients' quality of life. This may include working with primary healthcare providers such as general practitioners, family doctors, and nurses; physician specialists such as otolaryngologists and neurologists; and other allied health providers such as speech-language pathologists, optometrists, physical and occupational therapists, and others. Audiologists also work closely with professionals from non-healthcare settings, such as educators, social workers, and policy makers. However, despite audiologists' experience communicating with other providers, they continue to function primarily within their own specialty; essentially, audiologists often work in a "silo."
${ }^{1}$ Osborne College of Audiology, Salus University Elkins, Park, Pennsylvania.

Address for correspondence: Lindsay M. Bondurant, Ph.D., Osborne College of Audiology, Salus University, 8360 Old York Road, Elkins Park, PA 19027 (e-mail: lbondurant@salus.edu).
Humanitarian Audiology; Guest Editor, Steven P. Smith, Au.D.

Semin Hear 2020;41:92-99. Copyright (C) 2020 by Thieme Medical Publishers, Inc., 333 Seventh Avenue, New York, NY 10001, USA. Tel: +1(212) 760-0888. DOI: https://doi.org/10.1055/s-0040-1708506. ISSN 0734-0451. 
In considering improvements of healthcare delivery across specialties, the phenomenon of healthcare silos has been discussed as a key issue to be addressed. ${ }^{1,2}$ In this context, the term "silo" refers to the provision of healthcare within a specialty with limited communication and consideration of issues that relate to other specialties. The silo mentality in healthcare can cause divisions that negatively impact communication and processes, which then impacts the organization, the providers, and the patients. ${ }^{3}$ This is true for traditional healthcare models, and is increasingly being discussed for public health ${ }^{4,5}$ and humanitarian healthcare models. ${ }^{6,7}$ One avenue for breaking down silos may be by increasing interprofessional collaborative practice (IPCP), which allows healthcare providers and other professionals from a variety of fields to work together to provide more comprehensive service delivery, which can improve cost/benefit ratio and lead to better patient outcomes. ${ }^{8-10}$

The purpose of this article is to present a perspective on the potential applications of IPCP in humanitarian audiology. A conceptual framework for IPCP will be explored, followed by a discussion of potential avenues of partnership between audiologists and other healthcare providers working in the realm of humanitarian audiology. The goal is to begin a conversation about how audiologists can leave their silos behind, to participate in truly collaborative patient-focused projects, and to broaden their definition of interprofessional teamwork.

\section{WHAT IS INTERPROFESSIONAL COLLABORATIVE PRACTICE?}

The World Health Organization ${ }^{11}$ defines IPCP as occurring when "multiple health workers from different professional backgrounds provide comprehensive [clinical and non-clinical] services by working with patients, their families, carers and communities to deliver the highest quality of care across settings." While it is not a new conceptopportunities for care providers to work together have always existed in settings where healthcare is needed-there has been an emerging body of literature that suggests that IPCP initiatives can lead to better patient outcomes. The growing evidence indicating that IPCP can be instrumental in improving healthcare delivery and patient quality of life has led to efforts to create conceptual frameworks and guidelines for IPCP in a variety of settings.

In 2011, the Interprofessional Education Collaborative Expert Panel, ${ }^{12}$ including representatives from the professions of nursing, medicine, dentistry, pharmacy, and public health, published a set of core competencies for IPCP. The group suggested that to move forward with the best care possible, practitioners of IPCP should demonstrate competency across the four general domains described later. Within these domains, specific competencies are provided as a framework for IPCP teams to develop effective working relationships that lead to high-quality patient care in a variety of settings. Following is a summary of a few of the specific competencies within the four domains:

1. Roles/Responsibilities: Use full scope of knowledge, skills, and abilities of team members; recognize one's own limitations; and clarify each member's responsibility when executing treatment plans.

2. Communication: Plan for effective communication that supports the team's function; reduce or eliminate discipline-specific jargon; work to ensure common understanding of information and decisions; and consistent support of the team-based concept of IPCP for patient- and community-centered care.

3. Teamwork: Develop consensus on the roles, practices, and ethical responsibilities of the team and its individual members; engage other professionals as appropriate and integrate the knowledge and experience to make patient-care decisions; and share accountability for outcomes relevant to the healthcare provided by the team.

4. Values/Ethics: Prioritize patient-centered care; respect patient dignity and privacy; embrace and respect cultural diversity along with individual difference in patients as well as in the healthcare team; act with honesty and integrity; and maintain high standards of ethical conduct.

The core competencies presented by the Interprofessional Education Collaborative Expert Panel provide a general guideline for keeping a healthcare team focused on project 
characteristics that move humanitarian healthcare initiatives forward, always keeping the focus on the patient's needs. These guidelines can be used to develop a model for interprofessional collaboration in humanitarian settings. One such model will be discussed in the following section.

\section{A MODEL FOR INTERPROFESSIONAL COLLABORATIVE PRACTICE IN HUMANITARIAN HEALTHCARE}

Upon review of the domains outlined earlier, a possible framework for IPCP in humanitarian healthcare begins to emerge. In this framework, the domains of communication, teamwork, responsibilities, and ethics inform each step, as discussed below and shown in Fig. 1.

Step 1: Assessment of community needs. Ethical practice of humanitarian healthcare requires an assessment of the needs of the community that will be served. Due to the challenges of providing humanitarian outreach in underresourced areas, it may be difficult to collect information; therefore, in some aspects the needs assessment can be based on general population statistics regarding incidence and prevalence of various health issues, both acute and chronic. However, discussions with local providers and caregivers, along with community leaders, will be helpful in determining both the community's concerns, and also the likelihood of local participation in a humanitarian healthcare initiative. By including the local providers and community leaders from the beginning of the project, the likelihood of long-term investment is increased.

Step 2: Formation of the preliminary team. Based on the issues highlighted in the community needs assessment, a basic outline of an interprofessional team can be formed. As the team is developed, roles and responsibilities will need to be determined, and there should be general agreement on the project/team's values.

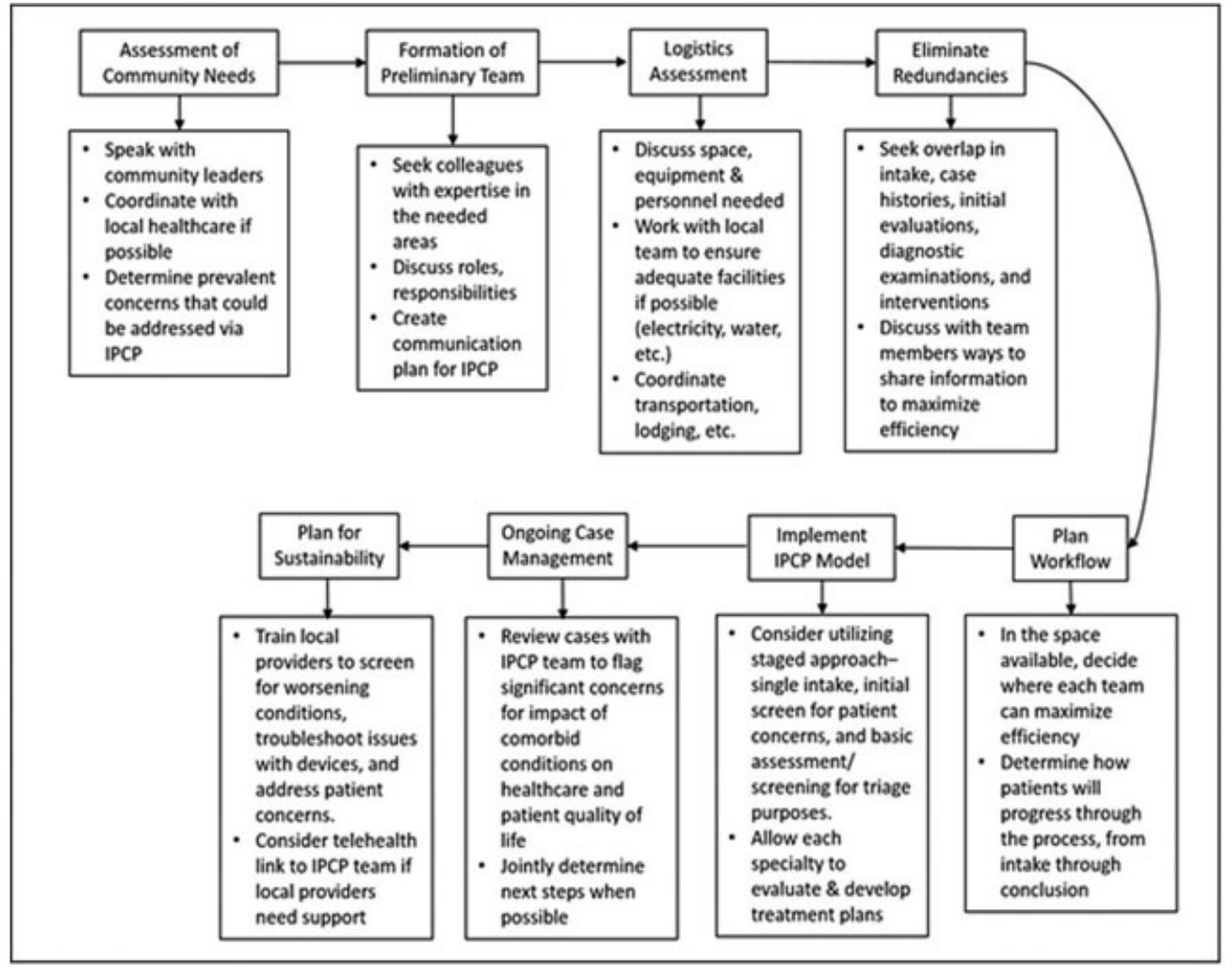

Figure 1 Model for interprofessional collaborative practice in humanitarian healthcare. 
At each step, members should refer back to the community needs assessment to determine how the addition or deletion of a given specialty will impact how the team will best serve the community's priorities. From a practical standpoint, some of the decisionmaking regarding the composition of the team will likely be based on the availability of various providers; however, all decisions should be focused on the shared goal of improving quality of life for members of the community to be served. Finally, as the team is being finalized, members should work on a communication plan for the project. The communication plan should include each member's contributions in terms of clinical and/or organizational specialty, and also an overarching plan to keep the care patient-focused.

Step 3: Logistics assessment. A key component of planning a humanitarian outreach program is an assessment of the logistics required to make the effort practicable. For any type of project, issues of location, accessibility, space, and utilities will need to be addressed. For an IPCP team, these issues may be compounded because the additional people and equipment will result in an increased demand for what is likely to be a limited supply of space and supplies. Having a thorough understanding of what each team member needs (both in terms of "bare minimum" and "ideal") will help in terms of acquiring adequate space and allocating supplies and utilities equitably between team members. Creativity may also be helpful-Can some exams be done outdoors while indoor spaces are being used by specialties with specific requirements such as dim lighting or specific structural elements? Can equipment be shared or scheduled for use at different times of the day depending on the team's needs? Once each specialty's requirements in addition to the requirements of the IPCP team are taken into account, along with the constraints of time, location, and accessibility, a clear plan can be developed.

Step 4: Eliminate redundancies. Individually and as a team, members will need to examine each step of the process to identify redundancies and consolidate processes whenever possible. Two key areas for this are intake and case histories. Instead of each specialty having patients provide demographic information (name, age, etc.), this could be centralized into a single paper or computerized chart. If there are overlapping case history questions (e.g., medication, health history, previous examination results), these could be consolidated into one document that could be filled out by an IPCP team member who is familiar with the project flow. While we never want patients to feel like they are on an assembly line, consolidation of basic processes and procedures can free up specialty providers to focus on the specific health issues they are trained to assess and address.

Step 5: Plan workflow. One of the last steps before embarking on the project is to plan the workflow to ensure maximum efficiency and to make the best use of team members' time. The work done on the previous steps should be extremely helpful in this stage. Advance planning (always with the knowledge that nothing ever goes completely according to plan) will help free up providers' time to effectively see as many patients as possible, and provide the patients with as many services as possible during the visit with the IPCP team. If the team is using a centralized intake area, space needs to be provided for initial screening and triage. This can help in determining where the patient should begin the healthcare journey with the IPCP team. The team members working on intake and other consolidated activities can create a plan to ensure proper flow to the correct provider. This will help reduce bottlenecks and facilitate communication between patients and the IPCP team. Having a specific space for patients to wait between exams and/or interventions can be helpful as it will free up space in the providers' areas. Each provider should have a clear plan for providing exams, documenting results, and providing treatment, counseling, or other interventions as needed.

Step 6: Implement the IPCP model. Once the planning is complete (again, keeping in mind that there will be things that do not go according to plan, so flexibility is key), it will be time to implement the IPCP model to provide humanitarian healthcare services in an underresourced area. Provider time can be preserved by including centralized screening and triage that can be completed by team members who are not completing exams or providing interventions at a given time. Furthermore, if team members who will be handling intake are also 
trained to take a case history flagging relevant risk factors, followed by basic functional and/or medical screenings, there may be a reduction in the number of patients who need to go through full exams, which would allow providers to provide focused care to the patients who need it most. Throughout the process, from intake through interventions, IPCP team members should strive to provide high-quality services while respecting each patient's dignity and unique needs and challenges.

Step 7: Ongoing case management. Communication among team members is a critical component of successful IPCP efforts. If possible, a team-based review of the patients seen each day is helpful for identifying comorbid conditions that may require specialized management plans. Coordinated case management plans can also streamline processes, avoid duplication of effort, and identify areas where patient safety may be at risk. For example, if multiple specialties recommend medication to manage various conditions, then a collaborative team meeting will help identify any potential interactions or other considerations, in addition to providing an opportunity to identify local resources for helping to maintain the treatment plan. Additionally, team-based case management and documentation can lead to cohesive recommendations to local providers, caregivers, and leaders, so that the IPCP's mission can be carried forward after the team's departure.

Step 8: Plan for sustainability. Any ethical humanitarian healthcare program, particularly those that address chronic conditions, should include plans for how patients and communities will be supported after the IPCP team has left the area. If device-based interventions were provided, is there a plan for maintenance, batteries/power supply, and repair? Is there an opportunity for local providers to assist patients, or for caregivers to be trained to continue the care plans, with the goal of maintaining the interventions provided by the IPCP team? For some communities, a well-thought-out IPCP plan can lead to the creation of a new job or career path for someone who has the desire and aptitude to continue the team's work. This is also an excellent opportunity to see if any type of telehealth options might be possible, allowing an opportunity for providers to stay in touch with the community to assist with troubleshooting and counseling.

This model is intended to provide some guidelines for planning an IPCP project, but the framework is by no means comprehensive or immutable. As anyone involved in humanitarian healthcare quickly learns, there is no "cookbook" approach that will work for every project, and a team must remain flexible to meet the needs of the community while working within the constraints of an under-resourced area. Starting a larger-scale IPCP project may seem daunting, but it does not have to be overwhelming. One approach may be planning an IPCP project from the beginning, with the team approach guiding the mission from its inception. Another approach could be expanding an existing project to include other professions. Either way, the guiding principle will always be the improvement of health outcomes and quality of life for the people served by the project, and with this at the forefront of the effort, the impact is more likely to be a positive one.

\section{INTERPROFESSIONAL COLLABORATIVE PRACTICE IN HUMANITARIAN AUDIOLOGY}

Audiologists know that hearing problems and/ or vestibular issues rarely-if ever-occur in a vacuum. Patients with hearing loss and/or balance problems often have other health issues that directly or indirectly impact the ability to diagnose and manage auditory/vestibular dysfunction. Conversely, patients' hearing loss can influence the ability to effectively understand and utilize the interventions being provided by other professionals. Finally, humanitarian outreach efforts by definition occur in areas where access to services is limited by financial, geographic, cultural, and/or other constraints. Given the poor uptake of interventions for hearing loss seen in well-resourced areas, ${ }^{13}$ it is likely that most of the communities served by humanitarian and public health efforts have issues with undiagnosed and unaddressed hearing and balance problems. As a result, audiology can be an integral part of an IPCP team in a humanitarian healthcare setting. This can happen in different ways in a variety of settings, some of which will be described below. 
Treatment and management of comorbid conditions. Hearing loss accompanies a variety of health conditions, including hypertension, heart disease, ${ }^{14-16}$ diabetes, ${ }^{17-19}$ and kidney disease. ${ }^{20}$ As a result, audiologists and other healthcare professionals may be able to leverage outreach efforts addressing these comorbidities to provide more comprehensive care. For example, humanitarian healthcare projects that include care for diabetes ${ }^{21}$ or cardiovascular disease $^{22}$ can and should include audiology services, to address the comorbid condition of hearing loss and to further improve patient quality of life.

Provision of preventative healthcare. Many humanitarian healthcare and public health projects focus on the provision of preventative healthcare, such as vaccinations and nutrition outreach, in nonemergent situations. These projects typically occur in communities experiencing poverty and poor access to medical interventions, and the recipients of these types of aid are likely to be experiencing other chronic healthcare issues. Audiologists and other healthcare professionals may be able to join a preventative healthcare effort to improve the impact of the services provided. As with projects focusing on the management of chronic health conditions, preventative health projects require coordination with local partners and the procurement of space and facilities. The inclusion of specialty care with a preventative care project would increase the value for all participants.

Identification and management of other disabling conditions. Humanitarian healthcare projects that focus on other sensory conditions and/ or issues that affect developmental trajectories are typically good fits for partnering with humanitarian audiology initiatives. Not only are higher rates of hearing loss found in people experiencing other sensory deficits, such as vision issues, ${ }^{23,24}$ but the general organization of these types of projects are similar to the setup required for audiology missions. The addition of audiology services will increase the likelihood that the community will see a positive impact on quality of life as a result of interventions provided by the team.

When proposing the inclusion of audiology on a healthcare team targeting other medical conditions, it may be helpful for the audiologist to highlight some of the following information:

- The incidence of hearing loss in the populations being targeted. ${ }^{25}$

- The impact of hearing loss on overall quality of life, including higher rates of depression, ${ }^{26-28}$ underemployment, ${ }^{29}$ and dementia in people with untreated hearing loss. ${ }^{30-32}$

- The increased cost of healthcare for people with untreated hearing loss. ${ }^{33}$

- The impact of untreated hearing loss on health outcomes, specifically as it relates to patients' understanding of case history questions, test instruction, and recommendations. ${ }^{34}$

Conversations with potential IPCP team members about partnering for a humanitarian healthcare project should adhere to the principles outlined by the Interprofessional Education Collaborative Expert Panel, ${ }^{12}$ with particular emphasis on ethics/values, communication, and teamwork. Each potential team member should recognize that IPCP projects allow for synergy through the collaborative process, and that the possibility of rewards that include more comprehensive service provision for patients, and improved quality of life for patients receiving additional care that otherwise would not have been received through a single-channel or "siloed" approach. Furthermore, the communities served by the project(s) may be further strengthened by sustainability plans that use a more holistic approach.

\section{CONCLUSION}

It has been long recognized that healthcare "silos" are suboptimal for patient care, and an emerging body of literature has highlighted the benefits of interprofessional collaboration in patient care. The application of IPCP principles to humanitarian healthcare, including audiology projects, is likely to improve outcomes for the patients seen in humanitarian initiatives in under-resourced areas. Although it may seem daunting to increase the scope of projects that are already rife with challenges, the effort is likely to result in improved quality of life for people in under-resourced areas. The potential for additional positive health outcomes beyond the scope 
of a "siloed" project should provide incentive to providers working in humanitarian healthcare.

\section{CONFLICT OF INTEREST}

None declared.

\section{REFERENCES}

1. Kelly YP, Goodwin D, Wichmann L, Mendu ML. Breaking down health care silos. Harv Bus Rev2019; digital articles: 2-4. Available at: https://hbr.org/ 2019/07/breaking-down-health-care-silos. Accessed October 1, 2019

2. Hajek AM. Breaking down clinical silos in healthcare. Front Health Serv Manage 2013;29(04):45-50

3. Meneses R, Caseiro J. Silos mentality in healthcare services. Paper presented at: 11th Annual Conference of the EuroMed Academy of Business, 2018; Malta

4. Bevc CA, Retrum JH, Varda DM. New perspectives on the "silo effect": initial comparisons of network structures across public health collaboratives. Am J Public Health 2015;105(Suppl 2):S230-S235

5. Sconyers R. The end of the silo: what it means for today's global health worker. 2015. Available at: https://www.devex.com/news/the-end-of-the-silo -what-it-means-for-today-s-global-health-worker -85224. Accessed October 1, 2019

6. Mirza M. Unmet needs and diminished opportunities: disability, displacement and humanitarian healthcare. New Issues in Refugee Research. 2011; 212. Available at: https:/www.unhcr.org/enus/research/working/4e0dbdb29/unmet-needs-diminished-opportunities-disability-displacementhumanitarian.html. Accessed October 1, 2019

7. United Nations. Amid unprecedented displacement, ending silos, funding shortages key to reducing suffering of millions, speakers tell economic and social council. Washington, DC; 2016. Available at: https://www.un.org/press/en/2016/ecosoc6775. doc.htm. Accessed October 1, 2019

8. Guck TP, Potthoff MR, Walters RW, Doll J, Greene MA, DeFreece T. Improved outcomes associated with interprofessional collaborative practice. Ann Fam Med 2019;17(Suppl 1):S82

9. Nielsen PE, Goldman MB, Mann S, et al. Effects of teamwork training on adverse outcomes and process of care in labor and delivery: a randomized controlled trial. Obstet Gynecol 2007;109(01): 48-55

10. Zwarenstein M, Goldman J, Reeves SInterprofessional Collaboration. Interprofessional collaboration: effects of practice-based interventions on professional practice and healthcare outcomes. Cochrane Database Syst Rev 2009;(03):CD000072
11. World Health Organization. Framework for action on interprofessional education and collaborative practice. Geneva: WHO; 2010. Available at: http://www.who.int/hrh/resources/framework_action/en/. Accessed October 1, 2019

12. Interprofessional Education Collaborative Expert Panel. Core competencies for interprofessional collaborative practice: report of an expert panel. Washington, DC: Interprofessional Education Collaborative; 2011. Available at:https://nebula.wsimg. com/3ee8a4b5b5f7ab794c742b14601d5f23?AccessKeyId=DC06780E69ED19E2B3A5\&disposition= 0\&alloworigin=1. Accessed October 1, 2019

13. National Institutes on Deafness and Other Communication Disorders. Use of hearing aids by adults with hearing loss: percent of persons with hearing loss who have ever used hearing aids. Washington, DC: NIH; 2014. Available at: https://www.nided. nih.gov/health/statistics/use-hearing-aids-adultshearing-loss. Accessed October 1, 2019

14. Chang TY, Liu CS, Huang KH, Chen RY, Lai JS, Bao BY. High-frequency hearing loss, occupational noise exposure and hypertension: a cross-sectional study in male workers. Environ Health 2011;10:35

15. Fisher D, Li CM, Chiu MS, et al. Impairments in hearing and vision impact on mortality in older people: the AGES-Reykjavik Study. Age Ageing 2014;43(01):69-76

16. McKee MM, Stransky ML, Reichard A. Hearing loss and associated medical conditions among individuals 65 years and older. Disabil Health J 2018;11(01):122-125

17. Bainbridge KE, Hoffman HJ, Cowie CC. Diabetes and hearing impairment in the United States: audiometric evidence from the National Health and Nutrition Examination Survey, 1999 to 2004. Ann Intern Med 2008;149(01):1-10

18. Horikawa C, Kodama S, Tanaka S, et al. Diabetes and risk of hearing impairment in adults: a metaanalysis. J Clin Endocrinol Metab 2013;98(01): $51-58$

19. Kim MB, Zhang Y, Chang Y, et al. Diabetes mellitus and the incidence of hearing loss: a cohort study. Int J Epidemiol 2017;46(02):717-726

20. Vilayur E, Gopinath B, Harris DC, Burlutsky G, McMahon CM, Mitchell P. The association between reduced GFR and hearing loss: a crosssectional population-based study. Am J Kidney Dis 2010;56(04):661-669

21. Miller AC, Arquilla B. Chronic renal insufficiency and diabetes mellitus following disasters: a model for reform. Prehosp Disaster Med 2007;22(02): s127

22. Friedland DR, Cederberg C, Tarima S. Audiometric pattern as a predictor of cardiovascular status: development of a model for assessment of risk. Laryngoscope 2009;119(03):473-486 
23. Chia EM, Mitchell P, Rochtchina E, Foran S, Golding M, Wang JJ. Association between vision and hearing impairments and their combined effects on quality of life. Arch Ophthalmol 2006;124(10): 1465-1470

24. Swenor BK, Ramulu PY, Willis JR, Friedman D, Lin FR. The prevalence of concurrent hearing and vision impairment in the United States. JAMA Intern Med 2013;173(04):312-313

25. Abrams H. Hearing loss and associated comorbidities: What do we know? Hear Rev. 2017. Available at: https://www.hearingreview.com/hearing-loss/hearing-loss-prevention/risk-factors/hearing-loss-associated-comorbidities-know. Accessed October 1, 2019

26. Hsu WT, Hsu CC, Wen MH, et al. Increased risk of depression in patients with acquired sensory hearing loss: a 12-year follow-up study. Medicine (Baltimore) 2016;95(44):e5312

27. Li CM, Zhang X, Hoffman HJ, Cotch MF, Themann CL, Wilson MR. Hearing impairment associated with depression in US adults, National Health and Nutrition Examination Survey 20052010. JAMA Otolaryngol Head Neck Surg 2014; 140(04):293-302

28. Mener DJ, Betz J, Genther DJ, Chen D, Lin FR. Hearing loss and depression in older adults. J Am Geriatr Soc 2013;61(09):1627-1629
29. Emmett SD, Francis HW. The socioeconomic impact of hearing loss in U.S. adults. Otol Neurotol 2015;36(03):545-550

30. Deal JA, Betz J, Yaffe K, et al; Health ABC Study Group. Hearing impairment and incident dementia and cognitive decline in older adults: the health ABC study. J Gerontol A Biol Sci Med Sci 2017;72 (05):703-709

31. Fritze T, Teipel S, Óvári A, Kilimann I, Witt G, Doblhammer G. Hearing impairment affects dementia incidence. An analysis based on longitudinal health claims data in Germany. PLoS One 2016;11 (07):e0156876

32. Heywood R, Gao Q, Nyunt MSZ, et al. Hearing loss and risk of mild cognitive impairment and dementia: findings from the Singapore longitudinal ageing study. Dement Geriatr Cogn Disord 2017; 43(5-6):259-268

33. Reed NS, Altan A, Deal JA, et al. Trends in health care costs and utilization associated with untreated hearing loss over 10 years. JAMA Otolaryngol Head Neck Surg 2019;145(01):27-34

34. Mormer E, Cipkala-Gaffin J, Bubb K, Neal K. Hearing and health outcomes: recognizing and addressing hearing loss in hospitalized older adults. Semin Hear 2017;38(02):153-159 